\title{
« Une vulgaire histoire de cul entre une putain et deux imbéciles »
}

\section{Nathalie Piegay-Gros}

\section{(2) OpenEdition}

1 Journals

Édition électronique

URL : http://journals.openedition.org/ccs/855

DOI : $10.4000 /$ ccs.855

ISSN : 2558-782X

Éditeur :

Presses universitaires de Rennes, Association des lecteurs de Claude Simon

\section{Édition imprimée}

Date de publication : 31 mars 2013

Pagination : 53-63

ISBN : 9782354121785

ISSN : 1774-9425

\section{Référence électronique}

Nathalie Piegay-Gros, « « Une vulgaire histoire de cul entre une putain et deux imbéciles » », Cahiers Claude Simon [En ligne], 8 | 2013, mis en ligne le 21 septembre 2017, consulté le 30 avril 2019. URL http://journals.openedition.org/ccs/855; DOI : 10.4000/ccs.855 


\title{
«Une vulgaire histoire de cul entre une putain et deux imbéciles ${ }^{1}$ "
}

\author{
Nathalie PIEGAY-GROS \\ Université Paris Diderot
}

Par cette remarque désabusée, Blum dégrade la tentation du romanesque : Georges peut bien continuer à «broder, à inventer des histoires, des contes de fées ", il butera toujours sur cette évidence : entre Corinne, de Reixach et Iglesia, ce n'était qu'une "vulgaire histoire de cul " et les beaux discours de Georges n'y peuvent rien. Georges l'entend bien, qui sait qu'ils ne sont eux-mêmes que des macchabées, encore, mais pour combien de temps, en vie. Pour autant, la machine à raconter, la dynamique du fantasme ne s'arrêtent pas. Georges veut comprendre comment de Reixach est mort, et avec lui, ce qu'est la force de destruction, la puissance de la mort à l'œuvre dans l'Histoire.

Quelle que soit la force avec laquelle le romanesque que contiennent les visions, les scénarios et les personnages (la femme $\mathrm{du}$ Conventionnel, Corinne, la fille vue dans la grange...) est rejeté, La Route des Flandres ne met pas la puissance du fantasme au service d'une écriture érotique qui se suffirait à elle-même. Le

1. La Route des Flandres, éd. Minuit, "double ", 1960, p. 174 ; Minuit « double", édition de référence : le titre du roman sera désormais abrégé en $R F$. 
désir d'histoires, et le désir de comprendre, de savoir, de restituer, est toujours présent. Mais le fantasme - désir de voir, d'avoir les femmes dont les soldats sont privés pendant la guerre et la captivité, mais aussi désir de savoir - déstabilise la représentation et le cours du temps sans pour autant délivrer la connaissance attendue. C'est que l'écriture de la sexualité, dans ce roman, en compromettant toute stabilité de la représentation, contrevient aussi à quelque forme que ce soit de figuration individualisante et psychologisante des personnages. Comme la guerre, l'acte sexuel bouleverse le rapport au temps - et donc le développement de l'intrigue romanesque. Le désir met en crise le récit et impose une nouvelle syntaxe narrative. Nous ferons l'hypothèse que c'est en dehors de ces cadres romanesques pourtant posés - le désir d'histoire, l'invention, l'affabulation - que Simon donne à penser le lien de la sexualité et de la guerre et celui du fantasme et de la réalité.

\section{Guerre et sexualité}

Pas plus que le roman n'oppose la violence destructrice de la guerre à la vitalité positive de la sexualité, il ne gratifie la réalité d'une supériorité par rapport au fantasme : lorsque le fantasme devient réalité - lorsque Georges peut voir et avoir Corinne pour de vrai il ne peut pas pour autant savoir. Dans la chambre d'hôtel où il fait l'amour avec Corinne, désormais veuve et remariée, Corinne à qui il a follement rêvé pendant cinq ans de guerre, Georges se remémore sa vie dans le camp de prisonniers et d'une façon plus générale, la destruction généralisée qu’a été la guerre. Il serait alors tentant de voir dans l'acte sexuel une sorte de résolution des conflits, un point d'aimantation des lignes centrifuges du roman, l'étreinte érotique faisant converger la dynamique de la mémoire et celle du fantasme $e^{2}$. Cette conception "harmonieuse " nous parait contredite par le fait que Georges, s'il cherche «à travers Corinne " $(R F, \mathrm{p} .278)$ à comprendre ce que peut être la destruction, la mort, la disparition, n’y parvient nullement. Lacte sexuel ne délivre aucun savoir : il

2. C'était le point de vue de Dominique Lancereaux, «Modalités de la description dans La Route de Flandres", Poétique, 1973, 14. 
mobilise l'énergie nécessaire pour savoir, pour se rappeler, pour rêver et continuer à désirer comprendre ; mais il ne figure ni ne représente de façon stable ce qui a été.

La Route des Flandres n'oppose d'ailleurs pas la sexualité et la guerre, comme on opposerait la vie et la mort, la construction et la déconstruction, la forme et l'informe. Tout l'imaginaire et l'esthétique de Claude Simon consistent au contraire à rapprocher les expériences de la guerre et de la sexualité. Comme on vient de le voir, d'abord parce qu'à travers Corinne quelque chose de la mort de Reixach, paradigme peut-être de la mort et la destruction en tant que telles, est recherché. Mais aussi parce que l'expérience sexuelle est fondamentalement archaïque, primitive, violente ; qu'elle ne donne prise à aucune forme stable, durable ; qu'elle est le contraire d'un ordre du temps et de la nature auquel on pourrait se fier. De même, elle n'est pas ce qui assure une identité que le temps, la mort, la guerre, la violence ont compromise, mais elle apparaît plutôt comme une pure énergie. Le montage à l'œuvre dans le roman multiplie les scènes où l'on passe sans transition de la violence de la guerre à l'expérience sexuelle 3 .

Car l'une et l'autre manipulent le temps, le précipitent ou le ralentissent, confondant les époques. On passe ainsi sans transition de la scène d'amour avec Corinne à la scène du fou que Georges entendait crier dans la grange ${ }^{4}$. Comme l'épigraphe de Malcolm de Chazal le laisse entendre, l'acte sexuel est ce qui devrait faire boire "le lait de l'oubli's", ce qui devrait permettre d'abolir le temps. Mais c'est plutôt à une sorte de perturbation massive du cours du temps qu'il aboutit : temps qui peut, dans l'amour comme dans la guerre, refluer, comme le mascaret, ou s'immobiliser, ou encore se dilater de manière étrange ${ }^{6}$.

3. Voir en particulier p. 242.

4. Voir p. 249.

5. P. 247. Voir l'épigraphe p. 239.

6 . Il reste que, comme souvent chez Simon, un correctif intervient pour souligner la nature illusoire de l'impression : « un instant seulement, ivres croyant que c'était toujours, mais un instant seulement en réalité comme quand on rêve que l'on croit qu'il se passe des tas de choses et quand on rouvre les yeux l'aiguille a à peine changé de place» $(R F$, p. 250). 
Cette perception du temps est rendue plus aiguë encore par le montage, qui déstabilise la linéarité : par le biais de calembours plus ou moins "vulgaires", pour reprendre le mot de Blum ${ }^{7}$ ou de syllepses jouant sur différentes significations des mots (" monter", " chevaucher ", " moule ", "gland "), les scènes sexuelles et guerrières se télescopent, se chevauchent, ce qui est raconté et ce qui est remémoré se confondant comme se confondent la mémoire et le fantasme.

\section{La bête apocalyptique}

Cette proximité entre les expériences de la sexualité et celles de la guerre apparaît clairement thématisée lorsque l'acte sexuel est présenté comme un " combat " $(R F$, p. 90). L'image du "bélier " est à cet égard particulièrement intéressante, associant la violence du registre polémique et l'animalité : "sourds, tous les deux tombés inanimés sur le côté mes bras l'enserrant toujours se croisant sur son ventre sentant contre moi ses reins couverts de sueurs les mêmes coups sourds, le même bélier nous ébranlant tous les deux " $(R F$, p. 276). Et quelques lignes plus loin : «nous étions comme une seule bête apocalyptique à plusieurs têtes plusieurs membres gisant dans le noir ». On passe alors immédiatement à l'évocation de corps de Blum et Georges dans la nuit du camp. Les " corps emmêlés " des amants sont ainsi associés aux cadavres ou aux corps des prisonniers : " c'est le fou, toujours maussade et morose il se recroquevilla essayant d'enfouir sa tête sous son manteau, [...] hurlant sans fin sans but dans les ténèbres, hurlant puis brusquement elle cessa dénoués nous gisions comme deux morts essayant sans y parvenir de reprendre notre souffle comme si avec l'air le cœur essayait de nous sortir par la bouche, morts elle et moi assourdis par le vacarme de notre sang " (RF, p. 249-250).

La sexualité est une expérience clairement associée à la mort ; c'est pourquoi, à travers Corinne, fantasmée pendant cinq ans et enfin possédée, Georges cherche à comprendre ce qu'ont pu être la destruction et la mort de son mari, de Reixach. On peut voir dans

7. Voir l'emploi du mot « con », p. 258. 
l'armoire de la chambre d'hôtel où ils se retrouvent le symbole de cette vanité et peut-être de cette morbidité de l'acte sexuel. Elle est destinée "à ne jamais rien renfermer sinon leur vide poussiéreux, poussiéreux cercueil des fantômes reflétés de milliers d'amants, de milliers de corps nus, furieux et moites, de milliers d'étreintes emmagasinées, confondues dans les glauques profondeurs de la glace inaltérable, virginale et froide » $(R F, \mathrm{p} .40)$.

\section{L'accointance immémoriale de l'homme et de l'animal}

Rapprochée de la folie ${ }^{8}$, l'étreinte sexuelle est aussi associée à l'animalité. C'est une constante de l'imaginaire érotique dans l'œuvre de Simon, comme le rappelle cet extrait de L'Acacia : « rejetant alors les draps, son corps mû à présent par quelque chose d'aussi furieux, d'aussi élémentaire et d'aussi impérieux que la faim ou la soif, retrouvant cette impétuosité (ou plutôt cette animalité) qui lui avait permis de ou plutôt l'avait forcé, en plein jour, dès que la sentinelle avait tourné le dos, à se jeter à quatre pattes dans le bois comme un chien, les mains déchirées, insensible $"$.

Dans La Route des Flandres, la représentation du sexe féminin est très fortement marquée par l'archaïque et l'animalité, l'œil auquel il est assimilé rappelant aussi la permanence dans l'imaginaire de Simon de la pulsion scopique : " un ovale partagé en deux et des rayons tout autour comme un soleil ou un œil vertical fermé entouré de cils et même pas de figure " $(R F$, p. 260). Est ainsi suggérée la continuité entre le désir de voir, le désir d'avoir et la volonté de savoir. Le sexe masculin, assimilé lui aussi à un œil ou à une bouche muette, est tout aussi archaïque : «à la fois bouche muette et oil furieux

8. Comme en témoigne la confusion des sensations sonores : "Roulant furieusement à droite et à gauche sur l'oreiller parmi la tache sombre de ses cheveux faisant Non Non Non Non ils avaient enfermé un fou dans la porcherie de la ferme en haut du pré qui leur servait de corps de garde, devenu fou dans un bombardement parfois ils se mettait à crier sans fin sans but semblait-il, paisiblement non pas tempêtant et tambourinant ou frappant contre la porte simplement il criait et quelque fois dans la nuit je me réveillais l'écoutant je dis Qu'est-ce que c'est, et lui c'est le fou ». ( $R F$, p. 249).

9. L'Acacia, éd. Minuit, 1989, p. 349. 
et mort aux bords rosis comme ceux de ces animaux poissons qui vivent dans les rivières souterraines les cavernes, devenus aveugles à force d'habiter les ténèbres bouche et oil suppliants et furibonds de carpe » $(R F$, p. 274). Les effets de gros plans pourraient rapprocher l'écriture de la pornographie, n'était la signification bien précisée qu'il faut porter à la description anatomique crue, sur laquelle insiste le texte : " les lèvres de la fente d'un bistre plus prononcé avant l'endroit où commence la muqueuse comme s'il persistait là mal effacé quelque chose de nos ancêtres sauvages primitifs sombres s'étreignant s'accouplant roulant nus violents et brefs dans la poussière les fourrés » $(R F, \mathrm{p} .260)$. Le détail cru ne se suffit pas à lui-même et ne vise pas à satisfaire le voyeurisme du lecteur : il est au service d'une interprétation, d'une vision de la sexualité. Elle est présentée comme une forme privilégiée non pas de remédiation ou de consolation - vision romantique que conteste tout le roman mais de résistance à l'Histoire, de tentative de sortie hors du temps, hors de l'Histoire. C'est en quoi la contestation du romanesque ni aventure, ni histoire d'amour - et la remise en cause de l'ordre et du cours du récit ont partie liée. L'association du sexe féminin à la/le " moule " est elle aussi révélatrice de ce frayage que risque Simon avec le discours le plus cru, pour évoquer une association du sexe féminin et du mal, dans un brassage de textes et de références mythologiques qui non seulement associe encore la guerre et le sexe, mais contrevient à toute forme d'individualisation et a fortiori de "psychologisation » de la femme ${ }^{10}$ :

cette bouche herbue cette chose au nom de bête, de terme d'histoire naturelle - moule poulpe pulpe vulve - faisant penser à ces organismes marins ou carnivores aveugles mais pourvus de lèvres, de cils : l'orifice de cette matrice le creuset originel qu'il lui semblait voir dans les entrailles du monde, semblable aux moules dans lesquels enfant il avait appris à estampiller soldats et cavaliers ( $R F$, p. 39-40).

Mais ce n'est pas seulement le revers de la rationalité que sexualité et guerre donnent à penser : ce sont aussi, et plus radicalement,

10. Je renvoie sur ce point aux analyses de Renée Ventresque, "Le trou et la trame : le sexe à l'œuvre ", La Route des Flandres, Claude Simon, Ellipses, "Capes/ agrégation ", 1997, p. 107 et suivantes. 
les limites de l'humain. Dans tout le roman, le bestiaire tisse un réseau qui rapproche expériences sexuelles et scènes de guerre. Les corps des amants relève du « devenir-animal » dont a parlé Deleuze. $\mathrm{Ce}$ " devenir-animal » dit la dimension régressive de l'acte sexuel : " J'étais un chien la langue pendante galopant haletant tous deux comme des chiens" ( $R F$, p. 275). Les emprunts à la mythologie accusent cette dimension "bestiale » de l'amour. Dans un premier temps, ils paraissent contribuer à dégager la relation amoureuse de toute approche individualisante et psychologique et renvoyer à un imaginaire collectif. Mais la spécificité de ces allusions - l'âne avec Apulée, dont on sait aussi l'insistance dans La Bataille de Pharsale, Léda et le cygne, Diane et son chien, Danaé ou encore Junon et le paon - est la manière dont l'antique est tordu vers l'archä̈que : il s'agit moins de figurer, donc de donner une image maittrisée de la pulsion, que d'évoquer la sexualité comme force essentiellement primitive. La référence mythologique n'est d'ailleurs pas plus exploitée pour son potentiel narratif - développements, métamorphoses d'un sujet et d'une histoire. Elle indique seulement l'accointance immémoriale de l'homme et de l'animal dans la guerre ou la sexualité. Une telle vision de la sexualité explique en partie le recours à des représentations elles-mêmes archétypiques, voire archaïques, de la femme. Dans la scène de la grange où les potentialités fantasmatiques sont si grandes, deux figures de femmes antinomiques sont décrites, l'une laiteuse et virginale, et l'autre, femme au profil de chèvre, qui est " comme la corruption, le châtiment même de l'idée de femme " $(R F$, p. 252). La femme vierge et impolluable d'un côté, de l'autre, celle que " chevauchent " les hommes et les soldats, une "salope " (le mot est employé par Iglésia, $R F$, p. 139). On pourrait discuter les valeurs morales, idéologiques, de tels stéréotypes. Mais c'est leur valeur de stéréotype en tant que tel qui nous semble ici importer : le fantasme a un pouvoir archaïsant, il renvoie à la production d'histoires et d'images, de scénarios qui pourraient être romanesques ou " anti-romanesques " (les "vulgaires histoires de cul "). Mais, dans le contexte de la guerre, il n'a pas le luxe de la nuance et du raffinement : il est la forme brutale, immémoriale de la pulsion, sans qu'il soit possible de nuancer, de spécifier. Corinne n'est-elle pas d'ailleurs, plus qu'elle-même, et si ce n'est une " fille à soldats " $(R F$, 
p. 260), la Femme ${ }^{11}$ ? C'est à ce pouvoir archaïsant (et généralisant) du fantasme que nous renvoient aussi ces images et ces mots sans nuance : loin de toute individualisation, de toute assignation à une identité déterminée, la mise en scène de la sexualité nous ramène à une vision archaïque de l'humain et de ses limites.

Cet usage du stéréotype et du mythe confirme que l'expérience sexuelle n'est ni un objet de représentation ni un matériau narratif - la Femme comme ce qui a lieu entre un homme et une femme sont réduits à des stéréotypes et la référence aux mythes n'autorise aucun développement narratif ni ne rehausse ce qu'il pourrait $\mathrm{y}$ avoir de trop cru, de trop vulgaire dans cette évocation : au contraire, le mythologique est lui-même dégradé vers l'archaïque, le romancier évitant soigneusement tout ce qui pourrait relever d'un imaginaire de l'antique érotico-solennel. Et pourtant, cette figuration de l'acte sexuel joue un rôle essentiel dans la constitution du roman et dans son développement : aliment principal de l'activité fantasmatique, elle produit des images, des scènes, des personnages ; plus, elle délivre une énergie - celle de la pulsion qui maintient en vie et pousse à savoir, puis à se rappeler - sans laquelle aucune enquête, aucune quête narrative ne serait possible.

Corinne, par exemple, ne révèle finalement rien d'autre qu'ellemême, que le plaisir qu'elle peut donner : " pensant après tout elle avait peut-être raison et que ce ne serait pas de cette façon c'està-dire avec elle ou plutôt à travers elle que j'y arriverais - mais comment savoir ?) peut-être était-ce aussi vain, aussi dépourvu de sens de réalité que d'aligner des pattes de mouche sur des feuilles de papier et de le chercher dans des mots, peut-être avaient-ils raison tous deux, lui qui disait que j'inventais brodais sur rien " ( $R F$, p. 278-279). L'analogie ici est très frappante, que le narrateur met en évidence, entre faire l'amour avec Corinne pour " savoir" et écrire pour "savoir ». L'un et l'autre peuvent être vains, c'està-dire ne délivrer aucune expérience fiable. Ecrire ou " connaître " Corinne, ce n'est pas réussir à connaître et à maîtriser ce qui a été, mais c'est continuer d'entretenir une relation fantasmatique avec la réalité - et donc à chercher comment savoir, à broder, à inventer, 
à écrire un roman, fût-ce contre le romanesque. Pas plus Corinne que l'écriture ne peuvent aider à comprendre ce qu'a été la mort de Reixach. Et réciproquement, lorsque Georges voit Corinne pour de vrai $(R F, \mathrm{p} .219)$, il ne parvient pas à lui raconter son expérience, pas plus que le narrateur de L'Acacia ne parle aux prostituées du bordel de Perpignan où il se rend un soir sur deux. La disjonction entre le fantasme et la réalité demeure intacte, mais le fantasme, désir d'écrire, de savoir, désir de voir, désir sexuels confondus, n’en est pas pour autant dévalorisé : c'est avec lui, à partir de lui que l'on écrit. Corinne, au demeurant, est certaine qu'elle n'est pour lui qu'une fille à soldats. Elle sait, ou croit, qu'elle ne coïncide pas avec celle que Georges dit aimer et avoir aimée pendant cinq ans. Par cette remarque, et la violence qu'elle traduit, Corinne insiste sur la discordance qui lui semble irréductible entre le pouvoir de l'imaginaire - où se conjoignent sans doute le récit, écrit ou oral, l'écriture et le fantasme sexuel - et l'ancrage dans la réalité. C'est en quoi la relation entre Georges et Corinne, et plus particulièrement la scène à l'hôtel, se distinguent si fortement d'un chapitre qui serait extrait d'une histoire romanesque entre deux personnages. Mais si la communication échoue, le besoin de raconter demeure.

Revenons au premier point de notre analyse : la vanité comparable de l'écriture et de la sexualité, si on cherche par leur " canal " une connaissance de la guerre, de l'amour, de la mort, qui soit plus efficace que ce que la puissance du fantasme autorise. La déception de Georges est emblématique de la faillite de la connaissance : quelque chose est voué à résister, à échapper. De même, le désir sexuel est voué à s'éteindre et à s'apaiser. C'est en quoi Simon se distingue fortement de Bataille ${ }^{12}$. Et passer du fantasme de Corinne au corps réel de Corinne n'y peut rien changer. Elle va libérer la mémoire de Georges qui, couché à ses côtés dans la nuit, va se rappeler la guerre et le camp. Mais pour autant, il ne prendra pas la place de Reixach, ne pourra faire coïncider fantasmes, corps et mots.

Lécriture s'alimente néanmoins à l'énergie de la sexualité; plus, les moments où le texte met en scène les fantasmes des personnages masculins bénéficient d'une sorte d'accélération des principes

12. Renée Ventresque l'avait justement noté dans son article, voir p. 112. 
moteurs de la narration : la syntaxe en est accélérée, syncopée, le texte bifurque encore plus rapidement et brutalement que d'habitude (de la nuit avec Corinne à la nuit du camp par exemple) et les mots enchaînent en les catapultant les scènes, faisant fi de toute continuité, la chevauchée des cavaliers et celle des amants se succédant sans transition aucune, par exemple ${ }^{13}$.

Le désir dans La Route des Flandres n'est donc pas seulement objet de représentation mais d'abord et surtout impulsion, flux qui aimante la langue; il défait la forme, l'arrache à une sédimentation, comme il défait l'identité. Le fantasme sexuel œuvre donc à la déstabilisation de la représentation et impulse à l'écriture une énergie mise au service du montage de fragments hétérogènes qui sont rapprochés comme les images psychiques se télescopent dans la mémoire de Georges faisant l'amour avec Corinne et revivant la guerre et la détention.

L'expérience sexuelle n'est donc pas le contrepoint positif de la morbidité propre à la guerre : on a vu qu'elle jouxte elle aussi la mort, la violence, le retour à l'archaïque. Elle n'est pas non plus ce qui propose une nouvelle forme qui résisterait à la destruction généralisée, au désastre qui gagne. Elle est le mouvement de cette forme, mouvement fait d'énergie, de force vitale, et de destruction de celle-ci, le désir étant voué à son extinction. La dimension pulsionnelle de l'écriture est force de liaison et de fragmentation mais aussi puissance de rupture. Elle aimante et fracture, fait surgir des formes, des corps, des scènes et les télescope avec d'autres, relie entre elles des fractions de temps séparés et discontinus. Elle n'est pas le contrepoint d'une écriture de l'Histoire, dont elle ne rémunère ni la violence, ni l'obscurité, ni la cruauté ; elle est une forme de résistance à l'Histoire, un de ces rares moments où le sujet

13. Voir en particulier p. 273 et suivantes, ou p. 244 : « je boufferais les pissenlits par la racine bouffant là où elle pisse suant nos corps emperlés exhalant cette âcre et forte odeur de racine, de mandragore, j'avais lu que les naufragés les ermites se nourrissaient de glands et à un moment elle le prit entre ses lèvres puis tout entier dans sa bouche comme un enfant goulu». 
peut croire qu'il amorce une sortie hors de l'histoire ${ }^{14}$. A ce titre, elle déjoue tous les codes et les attentes du romanesque. Mais ne pourrait-on in fine risquer l'hypothèse suivante : à un romanesque du sentiment, de l'histoire d'amour et de la stabilité de la représentation et des identités, fait place une écriture pulsionnelle, systématisant les ruptures et mettant en crise la narration. Du romanesque, elle conserve peut-être un " art d'assouvissement ${ }^{15}$ ", sans cesse déçu, mais suffisamment puissant pour qu'il relance sans cesse le désir d'écrire, de raconter, de savoir.

14. Voir Les Géorgiques, éd. Minuit, p. 119 et Dominique Viart, La Route des Flandres de Claude Simon, Une mémoire inquiète, Puf " écrivains », 1997, p. 223. 15. Selon le mot de Gracq dans En lisant en écrivant, Corti, 1980, p. 113. 